\title{
Magia parece, magnetismo es
}

\section{Enrique Vega Palas}

I.E.S. Carmen Laffón, C/. Alcalde Pepe Iglesias n 1, 41300 San José de la Rinconada, Sevilla, España. vegapalas@gmail.com

[Recibido en febrero de 2010, aceptado en abril de 2011]

\begin{abstract}
Se presenta un conjunto de experiencias basadas en la interacción magnética, a partir de las cuales se puede construir juguetes. Esta actividad se ha desarrollado con alumnos de $3^{\circ}$ y $4^{\circ}$ de E.S.O. los cuales han elaborado los juguetes y explicado su funcionamiento a sus compañeros.
\end{abstract}

Palabras clave: Magnetismo; Equilibrio; Levitación magnética; Principio de Arquímedes.

\section{It seems magic, but it is magnetism}

We present a set of experiments, based on the magnetic interaction, from which some toys can be made. This activity has been put in practice with students of 3rd and 4th of E.S.O. who have built the toys, explaining their operation to their schoolmates.

Keywords: Magnetism; Equilibrium; Magnetic levitation; Archimedes' principle.

\section{Introducción}

El magnetismo es uno de los fenómenos físicos que más nos sorprenden, debido a los efectos de atracción y repulsión que producen los imanes.

Un imán tiene dos polos, polo norte y polo sur. Si se enfrentan dos imanes con los mismos polos se repelen, mientras que si se enfrentan dos imanes con polos distintos se atraen. Es importante reseñar que cuando se rompe un imán no se puede separar los polos magnéticos, sino que aparecen dos imanes con sus dos polos.

En los experimentos de levitación magnética se produce la repulsión de imanes enfrentados por los mismos polos, por lo que es fundamental que los imanes estén perfectamente alineados y colocados, ya que el equilibrio estable es muy difícil. Esto se puede observar cuando intentamos empujar un imán con otro, pues el imán que se empuja intenta girar, para cambiar el polo de manera que se quede pegado al imán que lo está empujando.

A continuación, se van a explicar algunos experimentos sencillos relacionados con el magnetismo.

\section{Tren de levitación magnética}

Con este experimento se demuestra que los polos iguales de los imanes se repelen. Es muy similar conceptualmente al MAGLEV que se ha desarrollado en Alemania, Japón y Francia. La construcción de este tren está basada en un artículo que aparece en la referencia (Ciencia fácil 2010).

El funcionamiento del tren levitante se basa en la repulsión magnética que se ejercen polos magnéticos iguales, como se ilustra en la parte central de la figura 1. Es importante que la superficie que actúe como tren tenga poca masa. 


\section{Materiales necesarios:}

- Cartón piedra o cartón pluma.

- Imanes de ferrita de $48 \mathrm{~mm} \times 19 \mathrm{~mm} \times 5 \mathrm{~mm}$.

- Láminas de metacrilato transparentes.

- 2 muñecos.

- Un tablón de madera.
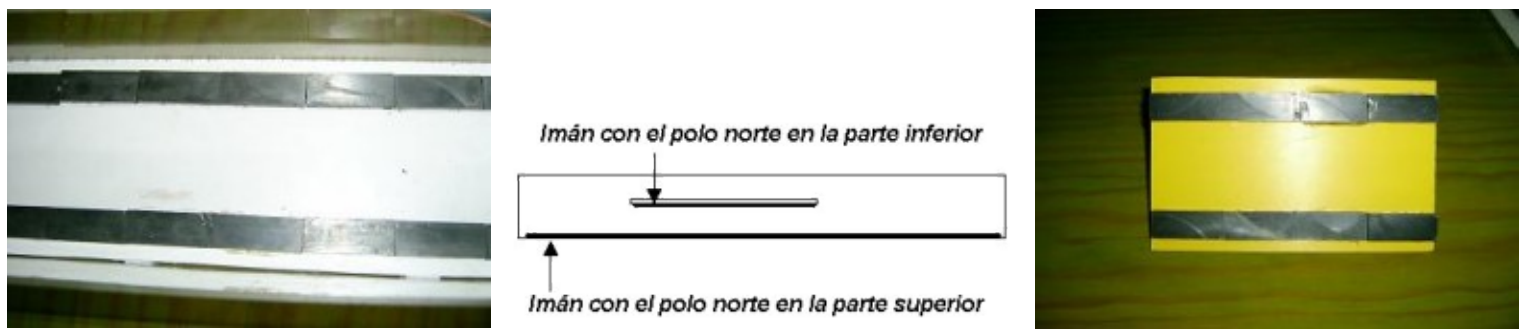

Figura 1. En el centro aparece el esquema del tren de levitación magnética. La imagen de la izquierda muestra los raíles formados por los imanes, mientras que la imagen de la derecha muestra la disposición de los imanes en la plataforma del tren.

\section{Procedimiento}

Los raíles se forman mediante los imanes de ferrita con su polo norte apuntando hacia arriba, los cuales se colocan a $1 \mathrm{~cm}$ de los bordes de una tabla o lámina de madera de unos $60 \mathrm{~cm}$ de largo y $15 \mathrm{~cm}$ ancho. Conviene poner en los lados y a los extremos de la tabla unas láminas de metacrilato transparente, de unos $15 \mathrm{~cm}$ de alto, para que veamos flotar al tren sobre los ráles, tal como se muestra en la parte izquierda de la figura 1.

La plataforma se construye con un trozo de cartón pluma no muy grueso, de $13 \mathrm{~cm}$ de ancho y unos $20 \mathrm{~cm}$ de largo. En la parte de abajo y a $1 \mathrm{~cm}$ de los bordes se colocan imanes de ferrita, como los de los raíles, que tengan la misma longitud que la plataforma. Es muy importante que los imanes estén bien alineados y que coincidan verticalmente con los de la base, tal como aparece en la parte derecha de la figura 1.

La clave para que el tren levite reside en colocar los imanes uno a continuación del otro de tal forma que se repelan cuando se vayan a pegar, pues si se pegan el tren no funciona. Esto se tiene que hacer tanto en la base como en la plataforma.

En la figura 2 se aprecia el tren, con un par de pasajeros, levitando sobre los raíles. Puede apreciarse las láminas de metacrilato y el tablón de madera que alojan en su interior el tren levitante. Puede verse videos y fotos de este experimento en el siguiente enlace: http://diariodeunexperimentadorcasero.blogspot.com/search/label/04 Feria de la Ciencia 2005.
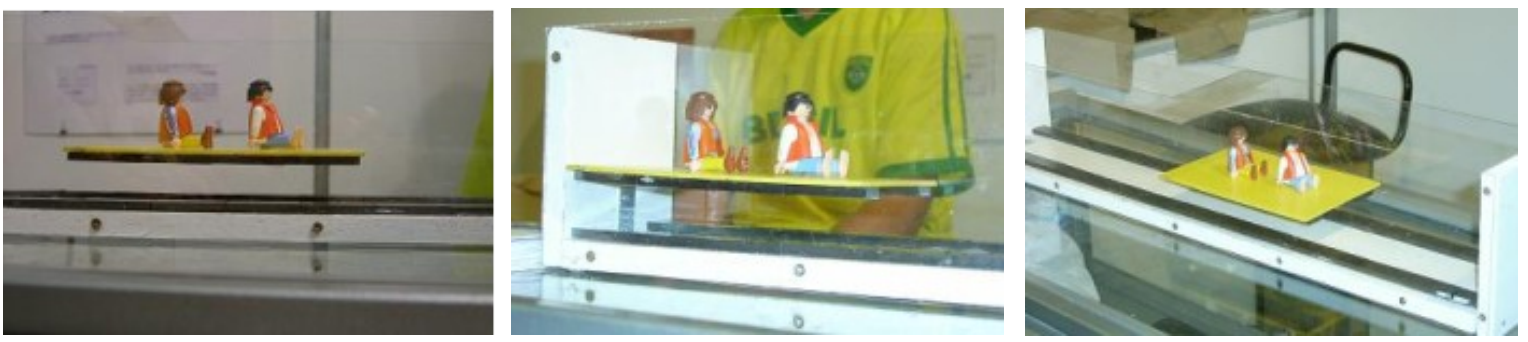

Figura 2. Imagen del tren funcionando, fotografiado desde diferentes perspectivas. 


\section{Escalador magnético}

Con este experimento pretendemos ilustrar el efecto de un campo magnético a través de una superficie y el principio de Arquímedes. Está inspirado en un juguete que aparece en la dirección que muestra la referencia (Grand Illusions 2010a).

\section{Materiales necesarios:}

- Tubo de PVC

- 2 tapones para tubo de PVC

- Un corcho de una botella de vino.

- 2 imanes, uno de neodimio de $5 \mathrm{~mm}$ de diámetro y $2 \mathrm{~mm}$ de alto, el otro es un trocito pequeño de un imán roto de ferrita.

- Un trozo de cartulina para hacer el muñeco.

\section{Descripción}

Consiste en un tubo de PVC, de unos $30 \mathrm{~cm}$ de longitud, en el que se introduce el corcho de una botella de vino con un imán pegado en su centro. Seguidamente se coloca uno de los tapones especiales para tubo de PVC y se llena de agua. Finalmente se coloca el otro tapón. En el exterior del tubo se coloca una figurita con un imán con el polo contrario al del corcho, para que se atraigan, tal como se aprecia en la figura 3.

El corcho se sitúa en la parte superior, porque flota. Cuando se invierte el tubo, el corcho tiende a subir arrastrando el muñequito de su exterior, produciendo

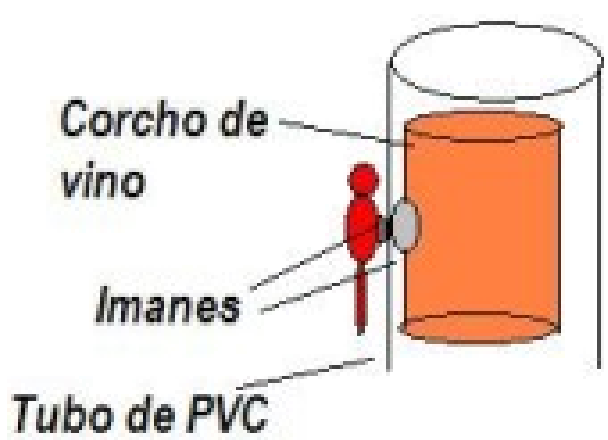

Figura 3. Detalles de los imanes pegados al corcho y a la figurita. un efecto muy espectacular.

En el funcionamiento del escalador magnético intervienen cuatro fuerzas. El empuje sobre el conjunto corcho-imán, el peso del conjunto corcho-imán, la fuerza de rozamiento entre los imanes y el tubo y, por último, la fuerza de atracción entre los imanes. Para que el muñequito ascienda por el tubo, la suma de la fuerza de rozamiento entre los imanes y el tubo y el peso del conjunto corcho-imán tiene que ser menor que el empuje sobre el conjunto corcho-imán. La fuerza de rozamiento (en módulo) es proporcional a la fuerza de atracción entre los imanes.

\section{Sugerencias para la construcción}

Es importante que el imán que está pegado al corcho sea potente pero no demasiado, ya que si es demasiado potente no permitiría subir al corcho. En nuestro proyecto hemos usado un imán de neodimio circular de $5 \mathrm{~mm}$ de diámetro. El imán exterior debe ser pequeño; en nuestro caso hemos usado uno de ferrita, que formaba parte de un trozo de un imán que se rompió.

Para sellar el tubo de PVC se ha empleado dos tapones de PVC pegados con un adhesivo apropiado. Otra opción es usar dos círculos de metacrilato transparentes para que se pueda ver el corcho, el cual se recubre con cinta adhesiva para que se empape de agua lo mínimo posible, aunque también se puede usar corchos de silicona. En la figura 4 se puede apreciar varios detalles del escalador magnético. 

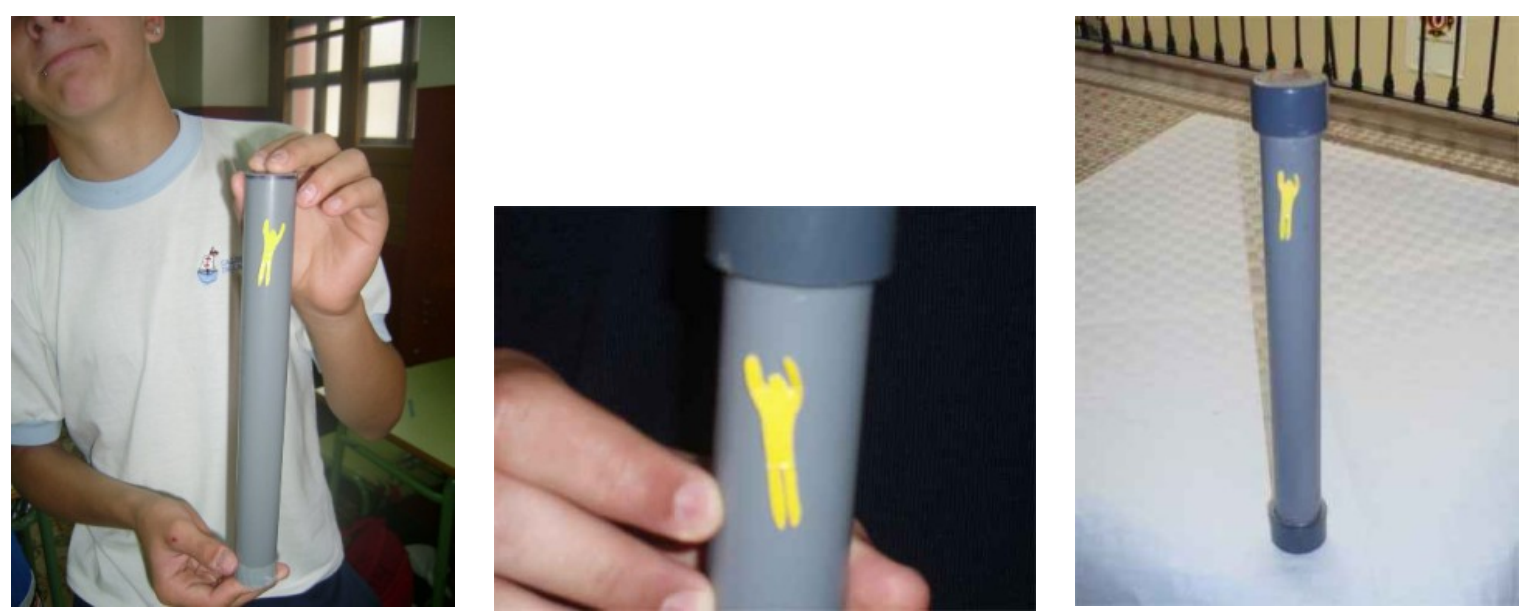

Figura 4. (Izq.) Un alumno muestra una imagen del escalador subiendo. (Centro) Detalle del muñequito, que está hecho con la portada de un cuaderno. (Der.) Otra imagen del escalador magnético, en la que se aprecia claramente los tapones especiales para tubos de PVC.

Para ver vídeos y fotos de este experimento se puede consultar el siguiente enlace: http://diariodeunexperimentadorcasero.blogspot.com/search/label/06 Feria de la Ciencia 2007.

\section{Pluma levitante}

Con este experimento se pretende demostrar el equilibrio existente entre la repulsión de los polos iguales de varios imanes y el peso de lo que hemos denominado pluma. El funcionamiento, cálculos matemáticos de las fuerzas que actúan y otros aspectos muy interesantes de la "pluma levitante" se pueden consultar en las referencias (Crane 1992, Kagan 1993, Ouseph 2006, Grand Illusions 2010b).

\section{Materiales necesarios}

- Imanes rectangulares de ferrita de $20 \mathrm{~mm} \times 10 \mathrm{~mm} \times 8 \mathrm{~mm}$.

- Imanes circulares de ferrita de diámetro externo $23 \mathrm{~mm}$, diámetro interno $4 \mathrm{~mm} \mathrm{y}$ altura $10 \mathrm{~mm}$.

- Un trozo de madera.

- 4 cuñas de madera con un ángulo aproximado de $45^{\circ}$.

- Una lámina pequeña de metacrilato.

- Un trozo de palo de pinchito de madera.

- Un envase de plástico cilíndrico de "lacasitos".

\section{Descripción}

Este juguete es útil para investigar las fuerzas que son capaces de sostener un objeto pesado levitando en el aire. Consiste en una pieza cilíndrica (envase de "lacasitos") que tiene imanes pegados en sus extremos, como se puede observar en el esquema de la figura 5, y que levita apoyada sobre una superficie de metacrilato sujeta a una base de madera, que también contiene varios imanes colocados de forma que la pluma no se caiga. La disposición de los imanes se puede observar en la figura 5 . 
Los imanes se colocan de dos en dos en los tacos de madera, en la posición en la que repelan al aro de ferrita que está pegado al envase de lacasitos, la repulsión debe ir en el sentido de la lámina de metacrilato. Hay que buscar el ángulo idóneo de los tacos de madera para que la pluma permanezca en equilibrio, en caso contrario los imanes tienden a atraerse. El ángulo es aproximadamente de $45^{\circ}$, como se puede observar en la figura 5 .

La pluma no cae porque los imanes están colocados con los polos iguales opuestos y se repelen. La lámina de metacrilato está situada de manera que la pluma no

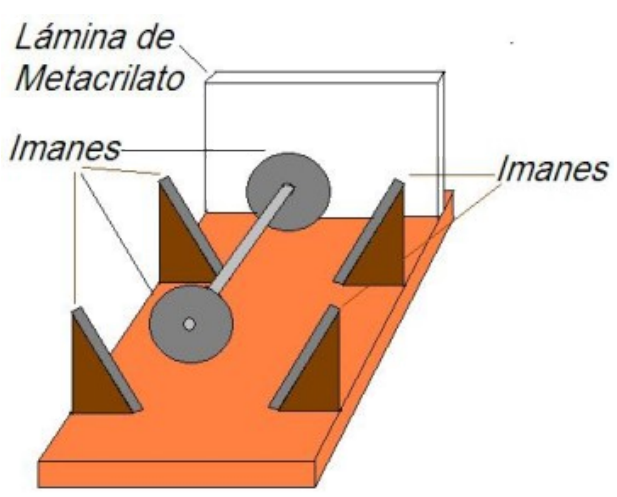

Figura 5. Esquema para la construcción de la pluma levitante. salga despedida hacia atrás. En la figura 6 se observa la disposición de los imanes y de la pluma levitando.

Los vídeos y las fotos de este experimento se pueden consultar en el siguiente enlace: http://diariodeunexperimentadorcasero.blogspot.com/search/label/06 Feria de la Ciencia 2007.
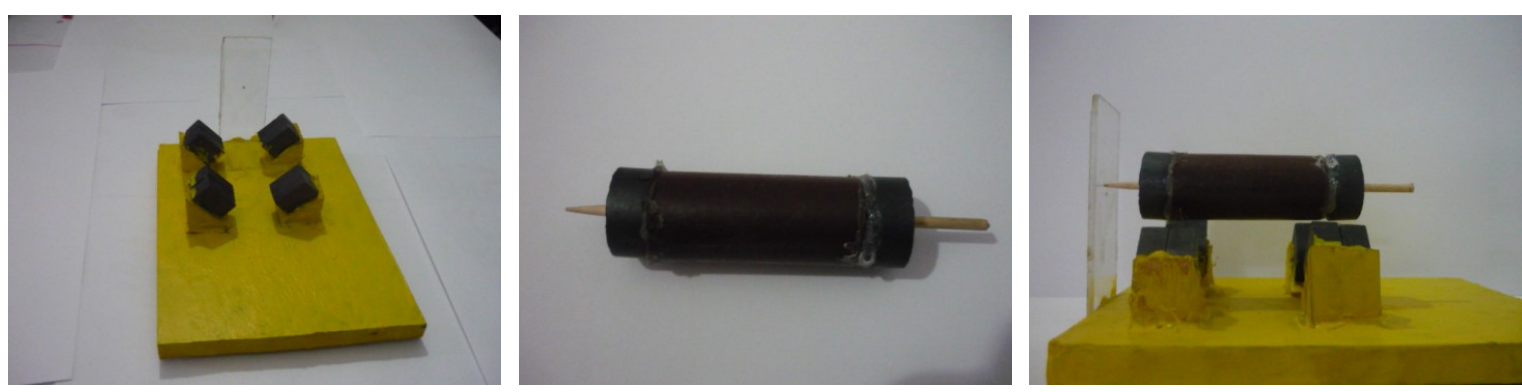

Figura 6. (Izq.) Disposición de los imanes en la base. (Centro) Pluma levitante. (Der.) Vista lateral de la pluma levitante en funcionamiento.

\section{Conclusión}

Estas experiencias fueron presentadas en la V Feria de la Ciencia de Sevilla, que se celebró los días 10, 11 y 12 de mayo de 2007. Estuvo enfocada a alumnos de $3^{\circ}$ y $4^{\circ}$ de E.S.O. (15-16 años) que no tenían conocimientos profundos sobre el tema, pues sólo habían estudiado un tema muy básico de magnetismo que se da en Física y Química en $3^{\circ}$ de E.S.O. Los alumnos respondieron muy bien, fabricando los diferentes experimentos y siendo posteriormente divulgadores en la caseta que teníamos en la Feria. En la evaluación que se realizó al finalizar la Feria los alumnos destacaron el aspecto positivo que presenta esta forma de aprender Física más dinámica, divertida y participativa.

\section{Referencias}

Ciencia fácil (2010) http://www.cienciafacil.com/paginamaglev.html

Grand Illusions (2010a) http://www.grand-illusions.com/toycollection/magic_stick/

Grand Illusions (2010b) http://www.grand-illusions.com/toycollection/revolution/

Crane H. R. (1992) Magnetic levitation (almost). The Physics Teacher 30 (Dec.), 540-541.

Kagan D. (1993) Building a magnetic levitation toy. The Physics Teacher 31 (Oct.), 432-433.

Ouseph P. J. (2006) Magnetic spinner. The Physics Teacher 44 (Dec.), 610-613. 